\title{
Use of Environmental Enrichment as a Clinical Methodology During Acute Experimental Infection by Trypanosoma Cruzi
}

\author{
Maria Carolina da Silva Sousa ${ }^{1}$, Maria Inês Doria Rossi ${ }^{1}$, Alexandre Andrade Evagelista ${ }^{2}$ and \\ Gabriel Melo de Oliveira ${ }^{2 *}$
}

${ }^{1}$ Institute of Science and Technology in Biomodel, Brazil

${ }^{2}$ Cellular Biology Laboratory, Oswaldo Cruz Institute, Brazil

*Corresponding author: Gabriel Melo de Oliveira, Cellular Biology Laboratory, Oswaldo Cruz Institute, Rio de Janeiro, Brazil.

To Cite This Article: Maria Carolina da Silva Sousa, Maria Inês Doria Rossi, Alexandre Andrade Evagelista, Gabriel Melo de Oliveira. Use of Environmental Enrichment as a Clinical Methodology During Acute Experimental Infection by Trypanosoma Cruzi. Am J Biomed Sci \& Res. 2021 - 13(4). AJBSR.MS.ID.001886. DOI: 10.34297/AJBSR.2021.13.001886.

Received: 㘹 June 21, 2021; Published: 眥 July 13, 2021

\begin{abstract}
One critial point in the Laboratory Animal Science (CAL) is the non-invasive evaluation of Animal Welfare (BEA). The detection (predictive or diagnostic) of the presence of suffering in mice when used for scientific purposes is a gap. The manuscrip aim to create a technical-scientific form to preditive presence of suffering in mice lab, based on the principle of Refinement of the 3Rs and BEA monitoring. Our methodology was based in usual environmental enrichment the objects, the trapeze. From these observations, our study hypothesis emerged, which was, the creation of the \#1 Trap prototype and the measurement through the daily counting of the number of trapeze use during 15 minutes. Experimental acute infection by Trypanosoma cruzi was experimental model in Swiss Webster mice. Our results described that, after division into four groups: [N] without infection and [Inf] mice infected and without treatment. The predictive statistical difference was observed on the $11^{\text {th }}$ day post infection (dpi) when compared ([N]: $72 \pm 18.5$ Events number $/ 15 \mathrm{~min})$ and ([Inf]: $32 \pm 13.1$ Events number $/ 15 \mathrm{~min})(\mathrm{p} \leq 0.05)$. The $21^{\text {st }}$ dpi was the most severe time point ([N]: $75 \pm 17.3$ and [Inf]: $11 \pm 5.1$ Events number/15 min, respectively) $(\mathrm{p} \leq 0.05)$. So, our results suggest the \#1 Trap prototype has predictive and monitoring capacity for experimental disease course. It makes it possible to prevent suffering and preservation of mice welfare during acute T. cruzi experimental model.
\end{abstract}

Keywords: Mouse; Animal Welfare, Non invasive Parameters; Environmental Enrichment

\section{Introduction}

Despite detailed studies on the genus Mus, or Mus musculus species, their genealogy, phylogeny, and historical aspects of the lineage origin currently used in the laboratory, including classical inbred lineages, the lack of documentation and scientific reports become imprecise in explaining exactly which species (or subspecies). It is known that the first lineage developed in the laboratory was DBA isogenic [1]. Outbred stock mice (nonisogenic) Swiss and CD-1are the best known colonies. Interestingly, its origin is related to 200 mice from the Pasteur Institute (France) by Amédée Borrel provided by an unknown person. These animals were provided to André de Coulon of the Anticancereux Romand Center in Lausanne (Switzerland) [2].
In 1926, Clara J. Lynch, an oncologist researcher of the Rockefeller Institute (New York) imported two male and seven female mice from André de Coulon. In the laboratory all animals derived from these mice were known as the "swiss" a nickname used by Lynch in her laboratory. Lynch also collaborated and gave some of his new mouse, of the "swiss" to other researchers, both internally and to other institutions [3].

In 1932, one of those researchers who received the "swiss" was Leslie Webster, who carried out susceptibility experiments to viral infection and distributed the animals to academics and commercial breeders, including Carworth Farms in the United Kingdom, which produced what is now called Swiss Webster [3]. 
In conclusion, mice lab or Swiss Webster is a complex and extremely interesting animal. All knowledge about this animal, be it about your life in the wild or in animal facilities, is essential for Welfare condition and the presence discomfort and suffering with fast recognition. According to the Brazilian legislation, it is imperative to know the biology, physiology and behavior of these animals in order to simulate their natural conditions in animal facilities [4]. Moreover, the behavioral characteristics knowledge of these animals, promote empathy between the handler and the animals. Which increased maintenance the laboratory mice with high life quality [5].

The aim of our study was to evaluate the effectiveness of an environmental enrichment (EE) object, the Trapezium, for the assessment of animal health quality during, mainly in biomedical tests. So, having a large experience in experimental infection by Trypanosoma cruzi in Swiss Webster mice, we decided to use this experimental model to evaluate the effectiveness of the new methodology [6].

In resume, The experimental model formed by Swiss Webster mice (18 to 20 grams) and T. cruzi Y strain (inoculum of $1 \times 10^{4}$ parasites $/ \mathrm{ml}$ via the i.p. route) is characterized by a relatively fast course of the experimental acute phase $[7,8]$. Infection by the infective blood form of T. cruzi, during the first week, starts its pathogenesis, however without blood parasites presence (parasitaemia). Standard model demonstrates the "parasitemia peak", that is, the maximum number of parasites in the bloodstream at the 8th days post infection (dpi). Around the 15th dpi, there is the so-called critical point of acute myocarditis, where there is the presence of parasitic replication and activated inflammatory response. So between the $15^{\text {th }}$ and $21^{\text {st }}$ dpi is the most clinically critical phase for the animal. Probaly due to the heart and cardiovascular involvement. Observed, change in body posture, prostration, piloerection and cachexia. In some cases, we can also observe other clinical signs, such digestive system alterations. The acute mortality expected arround $60 \%$ of animals occurs due to the severity of acute myocarditis and its cardiovascular consequences. However, $40 \%$ of infected animals survive. Between at 28-30 dpi, we have the characterization of the acute phase, with the absence of parasites in the blood, remission clinical symptoms and the beginning of the chronic phase [9].

Although other EE objects, such as the formation of nests [6] can be used as objects for evaluating the BEA in several lineages, we decided use of the Trapezium. The main reason for choosing this object were: a) From the age of 5 weeks of life (relative to the body weight to be infected by T. cruzi) Swiss Webster mice make intensive motor and exploratory activity and high use of the Trapezium; b) Easy observation animal use of the object and fast to count of the events number in the Trapezium use; and c) Mice with discomfort and suffering, showed hipoactive behavior and decrease motor and exploratory activity.

Finally, the main objective for the achievement of this manuscript how apply a technical-scientific methodology (without personal subjectivities) and able demonstrates, through statistical results, impairment of clinical mice conditions [10]. Creation of a non-invasive parameter adequate of evaluating groups mice and without manipulation. This initiative is highly important to avoid murine model suffering and pain during trials and the reseacher decision of the animal end point.

\section{Methodology}

\section{Animals}

24 ( $n=8$ per assay in triplicate) male Swiss Webster mice, 3 weeks old, from the Institute of Science and Technology in Biomodels (ICTB/FIOCRUZ) were used. The animals were received and adapted to environmental conditions and respective environmental enrichment object for five consecutive days. Each small polycarbonate cage, four individuals were housed. The mice were kept in the LBC/LITEB Experimental Animal Facility (Oswaldo Cruz Institute) under humidity (40 to 50\%), Temperature (20 to $22^{\circ} \mathrm{C}$ ), Air Exchange (estimated at 25 changes/hour) and Photoperiod of (12h day/12h night). The floor used wood shavings with exchange for twice a week. In addition, animals were offered filtered water and autoclaved feed ad libitum. All procedures described in this project are in accordance Brazilian Laws and ethical principles approved by CEUA/IOC License: L-010/18.

\section{Experimental Model:}

The mice were separated into different groups: [N]: Animals without infection and [InF]: Infected and untreated mice. Then on the day of infection, the animals showed the experimental model pattern of $20.0 \pm 1.8$ grams.

\section{Parasites and Infection}

The Y strain of T. cruzi was maintained by passage in vivo, and the trypomastigote forms were isolated from the bloodstream as previously described [7]. The parasites were resuspended in phosphate buffered saline (PBS) and counted in a hemocytometer. The total concentration of obtaining infective forms of T. cruzi was adjusted to $5 \times 10^{3}$ parasites $/ \mathrm{ml}$. In each test, the infection of each animal was performed with $200 \mu \mathrm{l}$ of this suspension $\left(1 \times 10^{4}\right.$ parasites/ml) by intraperitoneal injection (i.p.). Uninfected control mice received only $200 \mu \mathrm{l}$ of saline solution.

\section{Parasitological Parameters}

Parasitemia (parasites count in the bloodstream) was determined daily from 5 to $30^{\circ} \mathrm{dpi}$ using the Pizzi-Brener method (Brener, 1962). Mortality was noted daily and the cumulative 
mortality rate was calculated at $30 \mathrm{o}$ dpi. Body weight was assessed weekly (at $0,7,14,21$ and $30^{\circ} \mathrm{dpi}$ ).

\section{Non-Invasive Parameters}

\section{Food Consumption}

Feed/water consumption was measured weekly $(0,7,14,21$ and $30^{\circ} \mathrm{dpi}$ ). Food consumption was performed by the difference between the weight (grams)/volume (milliliters) offered (300 $\mathrm{g} / 300 \mathrm{ml}$ ) and that removed and calculated after 07 days in each cage. The estimate of individual consumption was calculated using the formulas: Cons $_{\text {total }}=$ weight or added volume - weight or volume, after 7 days. Then the individual consumption estimate was: Cons $_{\text {ind }}$ $=$ Cons $_{\text {total }} /$ number of animals per cage / number days.

\section{Prototype \#1 Trap}

Prototype \# Trap 1 was structured through the coupling of an EE object used in the routine of the Environmental Enrichment Program of the Animal Experimentation Center of the Oswaldo Cruz Institute. In our tests, all cages were prepared with the Trapezium to receive the animals and carry out a 5-day adaptation to the equipment, before starting the test.

The Trapezium was produced at COGIC/Fiocruz by Dr. Miguel Brück using stainless steel wire with a thickness of 2 millimeters (mm). It has dimensions of $15 \mathrm{~cm}$ in length and $5 \mathrm{~cm}$ in width, with the tips adapted to fit the lid of the cages (Figure 1).

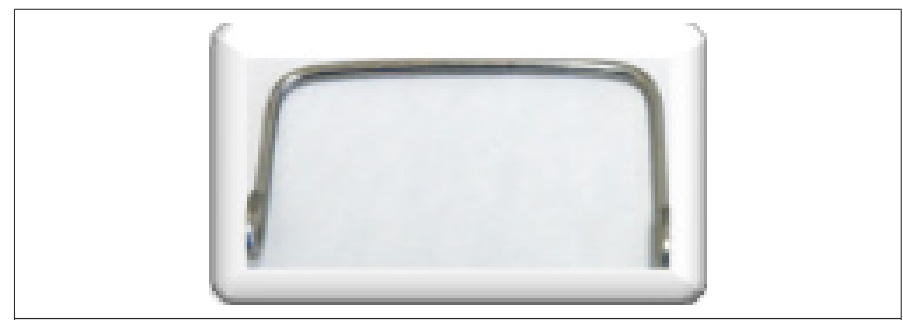

Figure 1: This is the Trapezium illustration that we used in the Oswaldo Cruz Institute's Environmental Enrichment Program. Source: Personal Archive.

\section{Trap \# 1 Protocol use}

The mice were received 5 days before the start of the tests. They were placed in their respective groups, in number 4 mice, after temporary individual marking (c1 to c4). We used a digital timer (Incoterm ${ }^{\circledast}$ - Brazil) and a Manual Counter (Starfer ${ }^{\circledR}$ - Brazil) to daily assess the number of mice times used the trapezium with the view through the cage side view per each group. The evaluation of the Trapezium use was performed using the following Ethogram: 1) Place both forelegs as support on the Trapezium; 2) Fully climb the Trapezium; 3) Jump over the Trapezium, with the fore and hind limbs touching the object; Was not counted (or included): Touching any part of the body on the trapezium, smelling the trapezium, going under or beside the object and others that were not mentioned above were not counted. In case of doubt, the count was not carried out. The Protocol was always applied at the same time (at 10:00 am) one hour after changing of the cage on cleaning days (Figure 2).

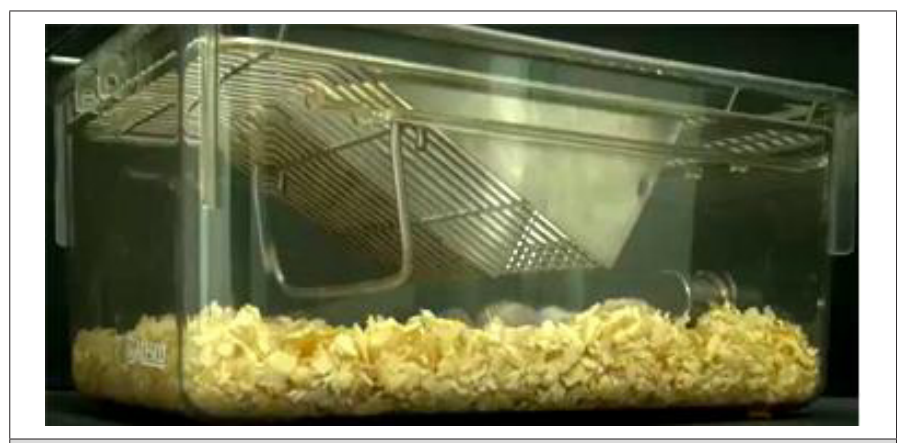

Figure 2: Illustration, in diagonal view, of the prototype \#1Trap for better understanding of the system. Source: Personal Archive.

\section{Record Tape}

Data collection was performed by filming outside the ventilated shelf by two portable cameras, with a frontal and lateral plane, using Sony HDR - PJ760 ${ }^{\circledR}$ and Sony HDR - XR550 ${ }^{\circledR}$ digital cameras.

\section{Statistical Analysis}

Initially, we will evaluate, using Excel and Prism, the sum, mean, percentage and standard deviation $( \pm S D)$ of the data obtained. We defined it as a significance factor $(\mathrm{p} \leq 0.05)$ under the Student $\mathrm{T}$ statistical test. These results will be confirmed by applying the Mann Whitney non-parametric statistical test with the same significance value.

\section{Results}

The results of the parasitological parameters (Figure 3) were similar to those described in the literature and in publications from our laboratory. The main parameter is the assessment and counting of the number of parasites in the blood (parasitemia) during the course of the acute infection. The parasitemia of the infected group [InF] were at the "parasitemia peak", that is, the point with the highest number of blood parasites, on the $8^{\text {th }} \mathrm{dpi}$, with values: $963.0 \pm 538.1$ parasites $\times 10^{4} / \mathrm{ml}$. In addition, showed a decrease in the parasites number, as expected, until the absence from the 26th $\mathrm{dpi}$, characterizing the end of the experimental acute phase and the beginning of the chronic phase (Figure 3A). Next, the important parasitological parameter is the animal cumulative mortality during the 30 days of infection. [InF] group, from the 14th dpi onwards, was the beginning of the animals' death (35\%) showed maximum level $(60 \%)$ from the $20^{\text {th }} \mathrm{dpi}$. The $40 \%$ population of surviving individuals, demonstrate a varied severity of clinical signs of infection. (Figure 3B). The parameter indirectly related to infection is the monitoring of the body weight of the animals in each group. In the weight curve. [N] animals demonstrated continuously growing, with the greatest weight gain, 20 and $30 \%$, between the 5th and 6 th week of the life [ $\left.7^{\text {th }} \mathrm{dpi}\right]: 21.3 \pm 1.4 ;\left[14^{\text {th }} \mathrm{dpi}\right]: 33.5 \pm 2.1$; 
[21 $\left.{ }^{\text {st }} \mathrm{dpi}\right]: 41.2 \pm 5.4$ and $\left[30^{\text {th }} \mathrm{dpi}\right]: 46.1 \pm 5.6$ grams. Infected animals that did not receive treatment $[\mathrm{InF}]$ showed the following weight curve: [7th dpi]: $32.0 \pm 2.3$; [14 ${ }^{\text {th }} \mathrm{dpi}$ ]: $28.4 \pm 5.8$; [21 ${ }^{\text {st }} \mathrm{dpi}$ ]: $16.8 \pm 2.9$ and $\left[30^{\text {th }} \mathrm{dpi}\right]: 33.7 \pm 5.3$ grams. The most clinically critical point of infection by the T. cruzi Y strain in Swiss Webster mice is suggested to be the $21^{\text {st }} \mathrm{dpi}$, with a decreased of almost $60 \%$ in weight body, when compared to uninfected animals (Figure 3C).
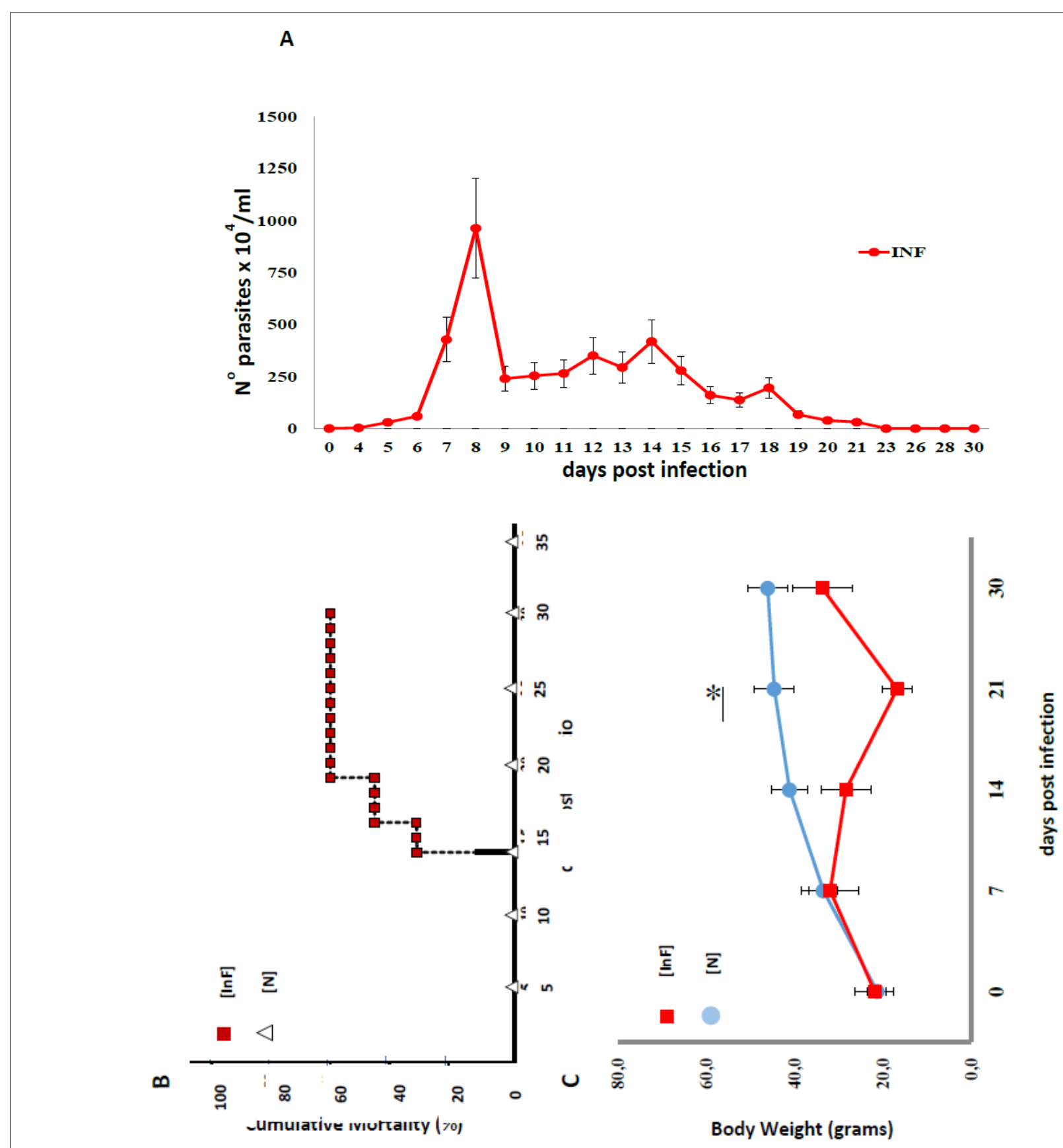

Figure 3: Parasitological parameters: Parasitemia of Swiss Webster \& Y strain in the T. cruzi acute experimental phase. Our experimental design consisted of two infected groups [InF], red square, and the group [InF], orange circle. Blood parasites were between the 1st day after infection (dpi) and up to the 30th dpi (A). Percentage of mortality of the experimental model (B). After the peak of parsitaemia (8th dpi) we observed the onset of mortality of $35 \%$ of animals on the 14 th dpi, increasing to $42 \%$ on the 16 th dpi and reaching its highest level on the 20 th dpi with $60 \%$ of deaths in the individuals of the $[\mathrm{InF}]$ group , red square. The $[\mathrm{N}]$ group, uninfected and untreated, white triangle, showed no mortality (B). Weighing the mice over the course of the infection demonstrated, again, that clinically the 21 st dpi is the most severe point of acute experimental infection by T. cruzi.. The groups of uninfected animals [N], white trianglem no alterations. However, the infection [InF], red square, at the 21st dpi promotes a drop of about $75 \%$ in the weight of individuals during this period (C). Total number of animals per group, including percentage of mortality, from 6 to 8 animals. The asterisk means the statistical difference, where $p \leq 0.05$ between the [Inf] and [N] groups through the Student T test and Mann Withney test. We express the \pm DP by the bars at each point. 
The respective experimental model continues to demonstrate BEA compromisse between the $14^{\text {th }}$ and $21^{\text {st }} \mathrm{dpi}$. We can consider the relationship of these results as compatible with the discomfort and suffering of the animals. In (Figure 4) we observe that there is a significant reduction in the spontaneous feed intake of infected mice [InF: $1.5 \pm 0.09$ grams] when compared mainly with healthy animals [N: $5.5 \pm 1.1$ grams]. The reduction in feed intake between $[\mathrm{InF}]$ and $[\mathrm{N}]$ is approximately $70 \%$. Water consumption is similar to feed consumption results. Infected and untreated mice [InF: $2.1 \pm 0.05$ milliliters] have a much lower water consumption than without infection [N: $8.1 \pm 1.6$ milliliters].
A

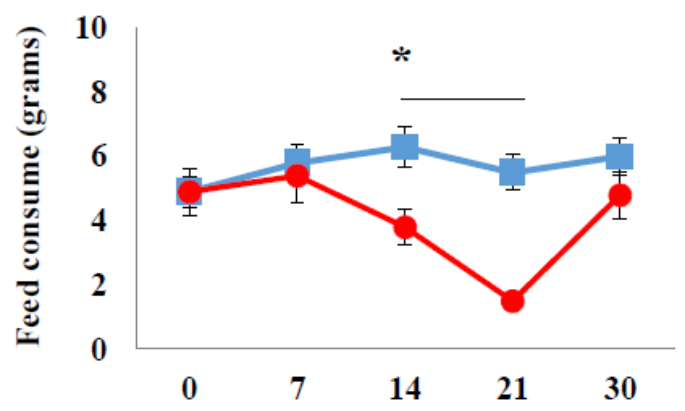

B

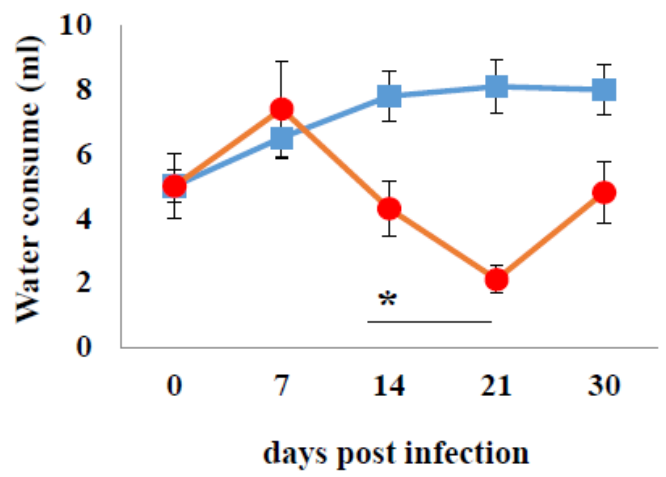

Figure 4: Food consumption: We measured the estimated individual mice's feed $(A)$ and water (B) consumption weekly during the course of T. cruzi infection. As previously described, we compared in groups [N] blue circle and [InF] red circle. Again, infection by T. cruzi during the acute phase in Swiss Webster mice promotes a clinical change between the $14^{\text {th }}$ and $21^{\text {st }} \mathrm{dpi}$, in this case the significant reduction in both feed and water consumption. T.cruzi infection promotes a severe drop in food consumption between the $14^{\text {th }}$ and $21^{\text {st }}$ dpi and the infected animals [InF] showed a significant drop $(p \leq 0.05)$ marked by the asterisk. Statistical significance calculated using Student's T Test and Mann Withney.

The Trapezium is an object used for environmental enrichment in mouse cages. In our routine we observed that sick animals, or with some kind of compromise in their harmonious social relationship, there was a decrease in the use of the trapezium. For this reason, we studied to structure a protocol that could be applied in experimental models and capable of predicting the onset of animal discomfort and suffering and/or diagnosing the animals that need to be removed from the trial and promoted end point (Figure 5).
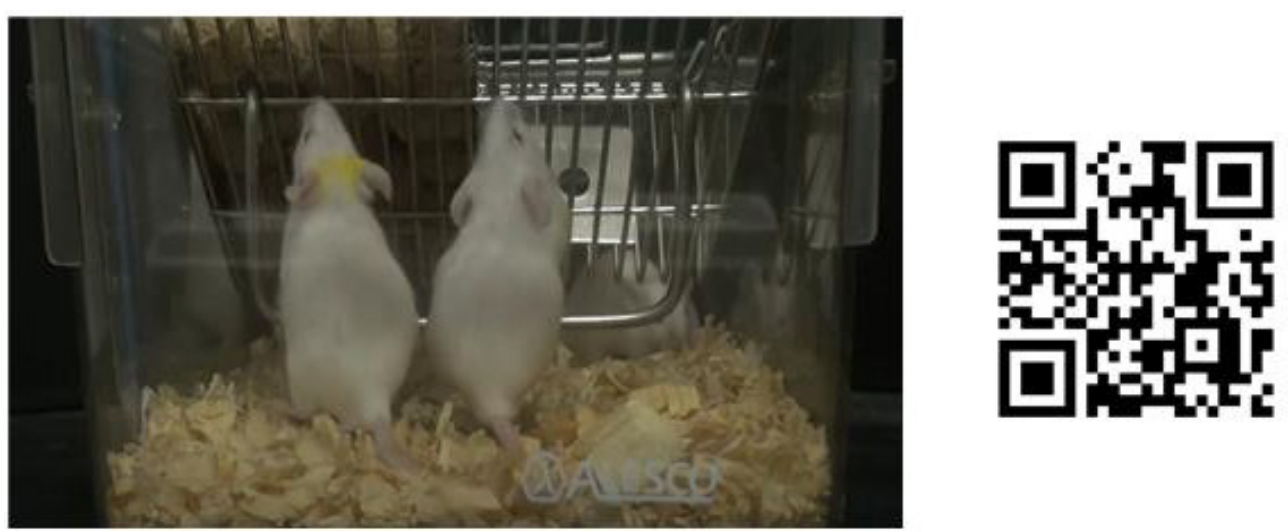

Figure 5: Illustration of mice without infection exerting a high interaction with the trapezius. Video available in QR code (QR code), on the right or.

Our results proved that our observations were technically and scientifically reproducible and reliable, despite the simplicity of the method. We propose to prototype \#1 Trap in a well-described experimental model, the Swiss Webster infected by the Y strain of T. cruzi (Figure 6). Our results demonstrate that before infection at dpi 0 , each group had similar values $([\mathrm{N}]: 31 \pm 3$ and $[\mathrm{InF}]$ :
$31 \pm 5$ Number of use of the trapeze/15 minutes). Up to $9^{\text {th }} d p i$ the values are increasing and similar. However, from the $10^{\text {th }} \mathrm{dpi}$ on, we started to observe differences between the groups. On the $10^{\text {th }}$ dpi the values were [N]: $104 \pm 12$ and [InF]: 80 No of events/15 minutes), however through the analysis statistics none of these differences were considered significant. However, at $11 \mathrm{dpi}$ the use 
of the Trapezium was able to provide values that were clinically imperceptible. The difference between the animals $[\mathrm{N}]$ and mainly [InF] were highly significant [N]: 116 \pm 11 and [InF]: $70 \pm 7$ Number of events $/ 15$ minutes) ( $p \leq 0.05$ ). This difference is aggravated during the acute infection course. At 15 th dpi there is a vertiginous decreased in the use of Trapezius by animals infected with $T$. cruzi [N]: $95 \pm 8$ and [InF]: $30 \pm 3$ Number of events $/ 15 \mathrm{~min})(\mathrm{p} \leq 0.02)$. The most serious period of infection, as reported in previous results, is in the third week of infection, and on the $21^{\text {st }} \mathrm{dpi}$, it shows the absence of Trapezium use by InF mice and the suggestion of severe discomfort and suffering [N]: $90 \pm 9$ and [InF]: 0 No of events 15 minutes $(p \leq 0,001)$. However, after animal mortality, we observed an attempt to recover the survival animals. [N]: $99 \pm 11$ and [Inf] $47 \pm 4$ Number of events/15 minutes.

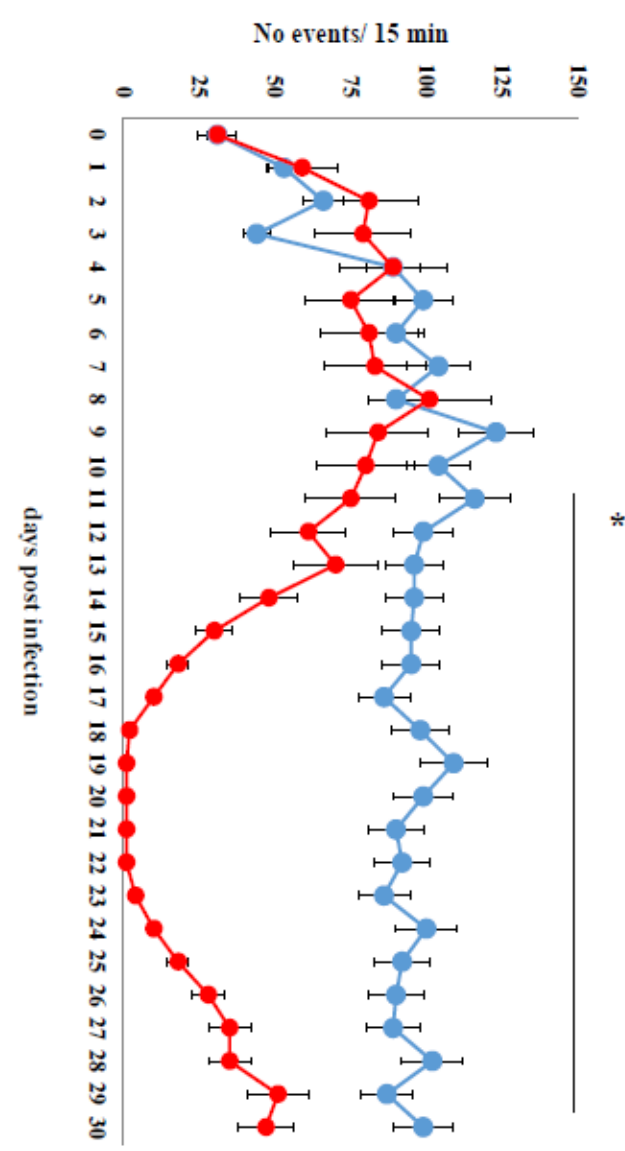

Figure 6: This figure is significant for the efficiency of the prototype \#1 Trap in monitoring the health condition of animals in experimental models, in our case, the acute chagasic. As already described, the group [N: blue circle] demonstrates that mice use the Trapezium constantly and continuously. However, the evolution of the disease, the presence of discomfort and suffering promotes hypoactivity to the animals, gradually losing interest in its use. Furthermore, it is directly related to health status, as animals surviving $T$. cruzi infection increase their interaction with the Trapezium [InF: red circle]. The asterisk illustrates a significant decreased between [Inf] and [N] ( $p \leq 0.001)$. Statistical significance calculated using Student's T Test and Mann Withney test. We express the $\pm \mathrm{DP}$ by the bars at each point.

\section{Discussion}

Our discussion will be based on the demonstration of the efficiency of the prototype \#1Trap in monitoring the BEA commitment of mice used for didactic or scientific purposes (experimental models).

Our first point is that so far, several studies have sought to assess the presence of discomfort, pain and suffering in mice during the trials. Most have a subjective character, performed by scores and by the criterion of the observed. One of the biggest examples is the Mouse Grimace Scale (MGS), where a manual is available (https:// www.nc3rs.org.uk/sites/default/files/documents/Guidelines/ MGS\%20Manual.pdf). Despite finding the use of this method valid and important, some critical points are evident:

i) Need and Training of a handler in the method and adequate infrastructure

ii) Use of cameras for filming and photography and the analysis can be carried out by the observer himself or through software

iii) Need for manipulation and isolation of the animal to perform facial photography

iv) Establish whether baseline MGS scores for mice are altered by other factors, for example when animals are dominant or subordinate [11].

The second point we would like to question is the classic test of corticosterone dosage as a direct relationship to the animal's discomfort and suffering. We believe that the interpretation of results is often completely wrong, in addition to being invasive in itself. The Mus mus musculus species, or as we believe, the Mus mus laboratorius, has a fight and flight system intrinsic to its preservation $[12,13]$. Then the stress response and adaptation system will be activated quickly, depending on the time and intensity of the stressful stimulus. Therefore, we must be careful when interpreting a single dose of corticosterone is a diagnosis of pain and suffering. We need to analyze that corticosterone itself has the capacity for negative feedback in the reaction axis to stressors, in short they would be:

a. Stressor factor;

b. Activation of the HPA axis with the release of CRF;

c. ACTH release and activation of the adrenal cortex and therefore the release of corticosterone that will act in various areas of the body, including the brain and neuromodulation in a stressful situation.

So everything depends on the relationship between three primary factors: 
I. As described above, intensity and duration of the stressor;

II. The animal's resilience and susceptibility to stress and, consequently,

III. If the stressor is within the animal's adaptive capacity, there will be a balance, which in the short medium term will tend to decrease serum corticosterone levels.

Therefore, the dosage of corticosterone, from our point of view, as a stress marker, will only be valid if:

i. In a situation characterized as stressful, with weight loss, hyper or hypoactivity, corticosterone levels remain low;

ii. The continuous elevation of corticosterone, demonstrating that the animal is not able to adapt to the stressful situation, whether due to the intensity of the stressor or its resilience to any type of stress induction [14].

At this point, the question is right, which is the differential factor in our study. The evaluation of the experimental model, with pathogenicity with a cardiac focus, but profoundly systemic and of a relatively long course [15], to the point that we can assess the animals daily, where the pathogenesis begins to affect the animal's health, or better, to promote impairment of the BEA and of your health condition?

Second prototype \#1 Trap at $11^{\text {th }}$ dpi. After the parsitaemia peak, we began to observe a decrease in the use of the trapezium, however at 11th dpi the difference between the animal without infection $[\mathrm{N}]$ and healthy and the animals infected with $T$. cruzi $[\mathrm{InF}]$ is statistically significant. Making a parallel between the pathogenesis (still not fully clarified) the pathogenesis of acute experimental infection by T. cruzi, the clinical symptoms of the animals and the use of the trapezium, we can make the following description:

In all groups, the use of the trapezium is something that promotes BEA to the animal, being an efficient environmental enrichment. After infection, animals [InF] undergo an acute kidney injury, but mild and transient, which does not promote clinical signs, but activates RAAS that after the peak of parasitemia will act together, aggravating acute myocarditis [16]. This picture can be observed, starting at the $11^{\text {th }} \mathrm{dpi}$ and a marked drop until the $15^{\text {th }} \mathrm{dpi}$, a period where the parasites are in their greatest multiplication activity in the cardiac muscle and the greatest response At this point, the question is right, which is the differential factor in our work [17]. The evaluation of the experimental model, with a pathogenesis with a cardiac focus, but profoundly systemic and with an immunological course, in an attempt to eradicate the infection. Then, from the association of cardiovascular compromise, with attempted compensation, and now the compromised heart, the mouse began to evolve into a clinical picture of cardiogenic shock, as observed by the absence of trapezium use between 18th and 23rd dpi and clinically cachectic effects [18]. of exacerbation of the immune response, mainly of TNF (called by Tarleton, 1988) as cachexin. So depending on the severity and susceptibility of the animal, mortality started at the 14 th dpi, however between the 16 th and 20th dpi are the periods of greatest severity, that is, exactly as demonstrated by the use of the trapezium [19]. close our discussion, convinced that, despite a simple methodology, it is highly efficient and can be better developed as a brazilian technology, affordable and most importantly capable of predicting during the course of a systemic pathology the moment at which the animal begins to present discomfort and pain and suggest based on data (values) the best time for a end point.

\section{Conclusions}

The mouse is an extremely interesting and complex species. There are 11 to 14 million people live with human beings and have adapted to the most varied situations possible. Physiologically, and naturally, and accelerated, ready for the classic "Fight or Flight Response", then having a different stress threshold than ours. In short, we believe that the objective of the mouse is to live in a group, have a territory, offer food and reproduce. The most stressful factors for these animals are the abrupt change of environment, change in the group (mainly in adult males) and lack of food, especially water.

We describe this paragraph to support that we believe in the development of equipment, materials, inputs aimed at the refinement for the use of mice in the laboratory. We do not question the replacement of animals with methodologies that provide the same results. However, most of the time, reduction as an ethical concept is like saying we don't need a solution because there is no problem. Unfortunately, this is the result of the lack of knowledge about the animal model, in this case the mouse.

Our main conclusions were:

a. The objective of the study was achieved with the development of prototype \#1 Trap, which showed efficiency and sensitivity to determine that the decrease in the use of the trapezium as an indicator, including a predictor, of the presence of discomfort, suffering and pain, in different degrees;

b. The prototype's simplicity does not interfere with its efficiency, and it can be used both in loco for observation of the researcher, as well as for filming. The difference between these two modes did not exceed 15\% difference, only when the animals, in groups, had high activity. However, with the evolution of the disease and the fall in the use of the trapezium, there was no difference between counting in loco or by filming the group of animals; 
c. Trap\#1 can be used daily, without handling the animals, totally non-invasively, without the need for individualization of the animals to perform the test and with the possibility of filming. Filming can provide further results such as: a) which individual in the group has clinical signs prior to the group; b) which individual is more severe during the course of the disease;

\section{References}

1. Morse HC (1978) Origins of inbred mice. Academic Press Inc, New York

2. Hedrich HJ (2012) The House Mouse as a Laboratory Model: a historical perpective. In: Hedrich HJ [Eds.,] The Laboratory Mouse. San Diego: Academic Press, p. 9-13.

3. Goldim JR, Raymundo MM (2019) Historical Aspects of Animal Research

4. da Costa SM, Rossi MID, Evagelista AA, Oliveira GM (2019) Origin, Phylogeny and Natural Behavior of Mice: What is Their Influence on Welfare During their Maintenance in the House Facilities?. Am J Biomed Sci \& Res 5: (5)

5. BRASIL. DECREE No. 6,899, OF JULY 15, 2009. It provides for the composition of the National Council for Animal Experiments Control CONCEA, lays down rules for its operation and its Executive Secretariat, creates the Register of Scientific Use of Institutions Animals - Ciuca, by regulating the Law $n$ the 11,794 , of October 8,2008 , which provides for procedures for the scientific use of animals, and other provisions.

6. Oliveira, Gabriel Melo de, Martins, Thais Veronez de Andrade (2017) Evaluation of preference for the type of environmental enrichment used by swiss webster mice in vivarium through the interconnected cage system (SGI). Revista da Sociedade Brasileira de Ciência em Animais de Laboratório, São Paulo, 5(1): 17-34.

7. Araújo-Jorge TC, De Castro SL (2000) Questões da doença humana para trabalho em modelos animais. In TC Araújo-Jorge, SL de Castro, Doença de Chagas: manual para experimentação animal, Editora Fiocruz, Rio de Janeiro, p. 17-19.
8. Coura JR, Borges-Pereira J (2010) Chagas disease: 100 years after its discovery. A systemic review. Acta Trop 115(1-2): 5-13.

9. Marinho CR, D’Império Lima MR, Grisotto MG, Alvarez JM (1999) Influence of acute-phase parasite load on pathology, parasitism and activation of the immune system at the late chronic phase of Chagas disease. Infect Immun 67(1): 308-318.

10. Meira WS, Galvão LM, Gontijo ED, Machado-Coelho GL, Norris KA, et al. (2004) Use of the Trypanosoma cruzi recombinant complement regulatory protein to evaluate therapeutic efficacy following treatment of chronic chagasic patients. J Clin Microbiol 42(2): 707-712.

11. Miller AL, Leach MC (2015) The Mouse Grimace Scale: A Clinically Useful Tool? PLoS One 10(9): e0136000.

12. Moriwaki K, Miyashita N, Yonekawa H (1985) Genetic survey of the origin of laboratory mice and its implication in genetic monitoring. In the Contribution of Laboratory Animal Science to the Welfare of Man and A nimals, edn. J Archibold, et al. pp. 237-247.

13. Sage RD (1981) Wild mice. Evol Theor 7(81): 39-90.

14. Balcombe JP, Barnard ND, Sandusky C (2004) Laboratory routines cause animal stress. .Contemp Top Lab Anim Sci 43(6): 42-51.

15. Oliveira G, Masuda M, Rocha N, Schor N, Hooper C, et al. (2009) Absence of Fas-L aggravates renal injury in acute Trypanosoma cruzi infection. Mem Inst Oswaldo Cruz 104(8): 1063-1067.

16. Oliveira GM, Silva T, Batista W, Franco M, Schor N (2009) Acute Trypanosoma cruzi experimental infection induced renal ischemic/ reperfusion lesion in mice. Parasitol Res 106: 111-120.

17. Leon JS, Wang K, Engman DM (2003) Captopril ameliorates myocarditis in acute experimental Chagas disease. Circulation 107(17): 2264-2269.

18. Oliveira GM, Alves MSC, da Silva DR, Batista WS, Schor N (2012) Cardio/ Renal interaction during the acute phase of experimental Trypanosoma cruzi infection: Angiotensin II, AT1 receptor, aldosterone inhibition and mortality rate. In press 2012, J Exp Integr Med.

19. Tarleton RL (1988) Tumour necrosis factor (cachectin) production during experimental Chagas disease. Clin Exp Immunol 73(2): 186-190. 